\title{
An adjoint method for the assimilation of statistical characteristics into eddy-resolving ocean models
}

\author{
By ARMIN KÖHL* and JÜRGEN WILLEBRAND, Institut für Meereskunde Kiel, Düsternbrooker \\ Weg 20, 24105 Kiel, Germany
}

(Manuscript received 20 February 2001; in final form 11 March 2002)

\begin{abstract}
The study investigates perspectives of the parameter estimation problem with the adjoint method in eddy-resolving models. Sensitivity to initial conditions resulting from the chaotic nature of this type of model limits the direct application of the adjoint method by predictability. Prolonging the period of assimilation is accompanied by the appearance of an increasing number of secondary minima of the cost function that prevents the convergence of this method. In the framework of the Lorenz model it is shown that averaged quantities are suitable for describing invariant properties, and that secondary minima are for this type of data transformed into stochastic deviations. An adjoint method suitable for the assimilation of statistical characteristics of data and applicable on time scales beyond the predictability limit is presented. The approach assumes a greater predictability for averaged quantities. The adjoint to a prognostic model for statistical moments is employed for calculating cost function gradients that ignore the fine structure resulting from secondary minima. Coarse resolution versions of eddy-resolving models are used for this purpose. Identical twin experiments are performed with a quasigeostrophic model to evaluate the performance and limitations of this approach in improving models by estimating parameters. The wind stress curl is estimated from a simulated mean stream function. A very simple parameterization scheme for the assimilation of second-order moments is shown to permit the estimation of gradients that perform efficiently in minimizing cost functions.
\end{abstract}

\section{Introduction}

Data assimilation in meteorology and oceanography with strong constraint variational inverse methods tries to find a particular solution of a dynamical system that best matches the observations in a certain time interval (Le Dimet and Talagrand, 1986). In oceanographic applications of this inverse technique, the idea that parameters can be determined from a steady oceanic circulation has so far played an important role for the design of the experiments. This view and the

* Corresponding author. Present affiliation: Scripps Institution of Oceanography, 8605 La Jolla Shores Drive, La Jolla, CA 92093-0230, USA.

e-mail: akoehl@ucsd.edu demands for assimilation techniques are changing. Results from assimilation experiments with lowresolution models suggest that models with much higher resolution are needed in order to find an oceanic state consistent with real climatological data (Marotzke and Wunsch, 1993; Schiller and Willebrand, 1995; Yu and Malanotte-Rizzoli, 1998). Additionally, a steady-state ocean is not in agreement with observations, which reveal variability on a wide range of space and time scales.

Applications of the adjoint method in highresolution models came into fashion particularly with the use of satellite altimeter data (Moore, 1991). Although using the adjoint method for state estimations with high-resolution models seems to be straightforward, there are substantial difficulties connected with applications in present-day 
models. In the meteorological literature the initial condition is mainly regarded as a parameter for optimization with the aim of predicting the flow for the time that follows the assimilation period. From this point of view assimilation time is limited by the predictability of the flow. For longer assimilation times exact initial conditions cannot be recovered (Tanguay et al., 1995).

The circulation simulated by eddy-resolving models is also subject to chaotic dynamics which restricts the applicability of the adjoint method to very short time ranges. For ocean models this range is of the order of a few months, but in order to estimate parameters (as opposed to initial conditions) a considerably longer time period is necessary (Schröeter et al., 1993). Increasing the period beyond this time is accompanied by reduced convergence rates or even divergence of the optimization which starts in the smaller scales first (Tanguay et al., 1995). This behavior is a consequence of the chaotic dynamics in high-resolution models (Gauthier, 1992). The failure of convergence during the search for an optimal solution is directly related to the characteristics of the cost function. When extending the period of assimilation, an increasing number of secondary minima of the cost function emerges ( $\mathrm{Li}, 1991$; Stensrud and Bao, 1992). Among the few methods trying to extend the limits of the adjoint method are the quasi-static variational assimilation algorithm (Pires et al., 1996) and an averaging method by Lea et al. (2000). The former tries to avoid the solution getting trapped in secondary minima by tracking the absolute minimum over progressively longer assimilation periods, whereas the latter filters the effect of secondary minima by averaging sensitivities over many initial conditions.

For linear dynamics the sequential Kalman filter produces the same state at the end of the assimilation period as variational methods, provided the error statistics are known (Thacker, 1986). In spite of this relation, sequential methods which are widely and successfully used for the assimilation in high-resolution models (Killworth et al., 2001; Fox et al., 1998) do not show obvious problems connected with the finite predictability of these type of models. Limitations through finite time predictability enter the Extended Kalman Filter (EKF) along with linearized equations that predict the error covariance. For computational reasons most applications of sequential methods do not include a prognostic calculation of the error and therefore avoid the unbounded error variance growth that Evensen (1992) found by implementing the extended Kalman filter with a highresolution QG model.

From a statistical point of view the picture of a steady ocean is not completely inadequate, since certain characteristic features of the ocean circulation remain steady or change at least on time scales much longer than the eddy time scale. Lorenz (1975) introduced the concept of predictability of the second kind that regards a response of statistical properties to a change in forcing. Palmer (1993) regards the climate as the attractor of a nonlinear dynamical system in a quasi-stationary regime. He added a forcing function to the Lorenz (1963) system and found that the probability density function (PDF) changes with the forcing. From this he concludes that a change in the PDFs will reveal the influence of the forcing. The present work will follow his footsteps searching for an adjoint method that involves only statistical properties such as mean values and variability. Arguments exist for the potential of predicting the ocean climate state in the presence of a chaotic subsystem (Griffies and Bryan, 1997) which can be explained by considering subsystems with different characteristic time scales (Boffetta et al., 1998). The idea is to constrain only statistical properties, in order to extend the limits of the adjoint method beyond the predictability limit and to allow the utilization of eddy-resolving models for state estimations in at least a statistical sense.

In Section 2 the idea for an algorithm is illustrated by regarding the behavior of cost functions that are based on statistical quantities. The method is described in Section 3. An application to a QG model is presented in Section 4. Concluding remarks and a proposal for a possibility to include higher order moments are presented in Section 4.4 and Section 5.

\section{Lorenz model}

In order to extend the variational method beyond the predictability limit, it is clearly not sufficient merely to define the cost function on the basis of statistical quantities. Relying on the ability to find a special trajectory, that optimally repres- 
ents the statistical constraints, the original formulation of the adjoint method would still fail. This will be demonstrated in this section. Starting with the standard formalism and regarding the cost function will illustrate the idea for an alternative approach.

The Lorenz (1963) model is a well known chaotic system and results from a spectral truncation of the equations that describe the RayleighBénard convection problem. In the chaotic limit the Lorenz model no longer corresponds to a physical system. However, it shares some fundamental properties of real but more complex chaotic systems. The Lorenz equations

$\dot{x}=-\sigma x+\sigma y$,

$\dot{y}=\rho x-y-x z$,

$\dot{z}=-\beta z+x y$,

are integrated with a standard fourth-order Runge-Kutta scheme with a step size $\Delta t=0.01 ; \sigma$ is the Prandtl number, $\beta$ is the domain aspect ratio, and $\rho$ is a normalized Rayleigh number which is chosen as the control parameter in the following.

In order to demonstrate how invariant properties of the attractor could be used for the estimation of parameters, a cost function

$J=\left(\bar{x}-\bar{x}^{\circ}\right)^{2}+\left(\bar{y}-\bar{y}^{\circ}\right)^{2}+\left(\bar{z}-\bar{z}^{\circ}\right)^{2}$,

based on the mean position

$\bar{x}=\frac{1}{t} \int_{0}^{t} x\left(t^{\prime}\right) \mathrm{d} t^{\prime}$

of the trajectory is chosen where the reference values $\bar{x}^{\circ}, \bar{y}^{\circ}, \bar{z}^{\circ}$ are taken from experiments with the classical parameters $\sigma=10.0, \rho=28.0$, and $\beta=8 / 3$. The trajectory orbits the two unstable fixed points, $z=\rho-1, x=y= \pm \sqrt{\rho-1}$ and is moved when the parameter changes. The mean values are $\bar{x}=\bar{y}=0$ and $\bar{z} \simeq \rho-1$. The macroscopic sensitivity of the cost function with respect to $\rho$ that can be derived from finite perturbations can therefore be expected to approach $2\left(\bar{z}-\bar{z}^{\circ}\right)$ for long integration periods. The time dependence of the exact cost function gradient, $\partial J / \partial \rho$, as calculated by the adjoint is displayed in Fig. 1 and shows in contrast to this an almost exponential growth. This behavior is generic and due to the chaotic nature of the Lorenz system. Chaotic systems may be characterized by Lyapunov expo-

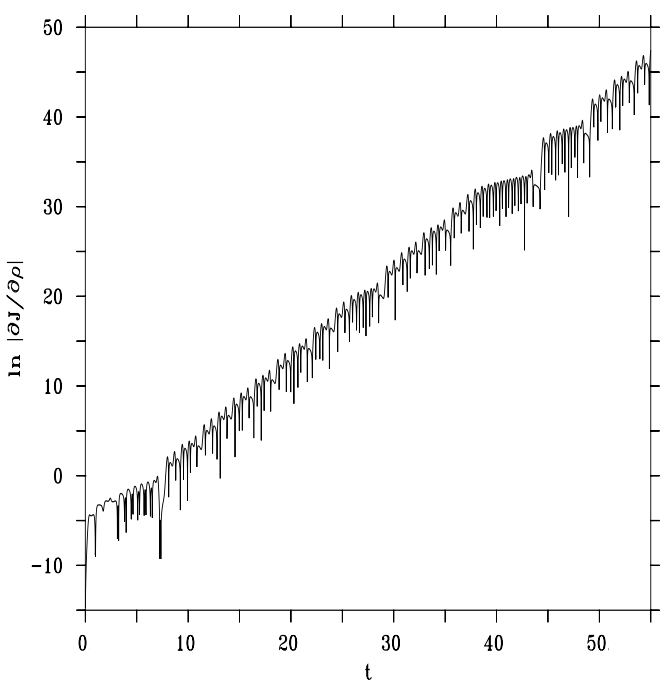

Fig. 1. Temporal evolution of the norm of gradient $\partial J / \partial$ $\rho$ of the cost function (4) calculated with the adjoint method. The Rayleigh number is $\rho=31$. The slope corresponds to a Lyapunov value of about one as calculated by Nese et al. (1987).

nents which give a quantitative measure of chaos (Oseledec, 1968). Positive values describe the sensitivity to small changes in the initial conditions. For $\rho>\rho_{\mathrm{H}}=24.74$ the Lorenz system is chaotic with Lyapunov exponents of $(0.93,0,-14.60)$ for the classical parameters (Nese et al., 1987).

Eigenvalues of the linearized and adjoint models are closely related to Lyapunov exponents and sensitivity analysis (Palmer, 1996). The mode of the adjoint model with the largest real part of the corresponding eigenvalue is the largest singular vector at initial time. In the limit of infinite time periods the largest Lyapunov exponent corresponds to the real part of the dominant eigenvalue of the modal growth of the adjoint model. The adjoint variable is dominated by the most unstable eigenvector of the adjoint propagator and therefore shows exponential growth.

The exponential growth in Fig. 1 corresponds very well to the maximum Lyapunov exponents calculated by Nese et al. (1987). Since the adjoint variable mediates the gradient of the cost function with respect to any parameter, exponential growth of adjoint variables is transferred to an exponential growth of almost all gradients. This is an expression for the fact that almost any parameter change 
causes a disturbance of the trajectory which leads to exponential error growth. Note that even in cases where the limit of validity of linearization is by far exceeded, the validity of the gradient obtained from tangent linear adjoint equations by the variational formulation still holds. The exponential growth must thus be related to characteristics of the cost function.

In physical systems state variables and cost function values are normally bound by an upper limit. Since exponentially increasing cost function gradients are generic for chaotic systems (appearing for almost all parameter choices rather than only for a certain set of parameter values), they are necessarily accompanied by a growing number of secondary minima and vice versa. The existence of secondary minima that prevents the convergence of the adjoint method can therefore be diagnosed from an exponential increase of the norm of the adjoint variables. In the limit of long integration times, sensitivities estimated by the adjoint method only reflect the chaotic nature of the model and are different to sensitivities that were derived from finite perturbations of model parameters in sensitivity studies. However, the chaotic nature of high-resolution models also blurs the assessment of a finite perturbation used in sensitivity studies in the way that a reliable influence of the parameter is only detectable if the perturbation is large enough or the integration time is long enough. Otherwise it is impossible to separate the effect of slightly changing the attractor from a macroscopic change of the trajectory on a nearly unchanged attractor. Small perturbations cause a macroscopic change in the trajectory but in general a smooth change of the underlying dynamics expressed by the attractor (Eckmann, 1981). An exception from this is bifurcation points, where the topological nature of the attractor may change when the parameter crosses certain critical values. For sensitivity studies with ocean models in particular, a different model result may suggest the influence of a changed parameter, whereas the true reason lies in a different realization of the chaotic eddy field that is sensitive to any changes. It is therefore necessary to provide error information with any quantity derived from an eddy resolving model that gives an idea how large the influence of different realizations of the eddy field on this quantity is.
The evolution of the cost function (4) in dependence on the parameter $\rho$ is shown in Fig. 2 for a number of increasing integration periods. For small intervals $t$ the cost function seems to have a unique minimum and is significantly non-parabolic due to the nonlinearity of the system. Extending the interval beyond a few times the characteristic time scale is accompanied by the emergence of an increasing number of secondary minima. The characteristic time scale (of order of one in the Lorenz case) might be compared to the eddy time scale of one month for ocean models. In the limit of very long integration times the cost function approaches a parabolic form, reflecting the almost linear dependence of the position of the attractor on the parameter $\rho$. Secondary minima are no longer resolved and appear as stochastic deviations. This stochastic limit that is approached for $t=200$ in the Lorenz case would correspond to an integration period of the order of a decade for ocean models.

The Lorenz model is deterministic so that the cloud of dots still belongs to a continuous curve. However, a detailed investigation of the distribution of mean and cost function values from a small interval around $\rho=31$ shown in Fig. 3 reveals a Gaussian distribution for the mean values. This result suggests that statistical moments of the nonlinear Lorenz model might be described by a less complex model (e.g. $\bar{x}=\bar{y}=0$ and $\bar{z} \simeq \rho-1$ ) plus stochastic white noise forcing with variance taken from Fig. 3.

For infinite integration times the cost function as in Fig. 2 will approach a curve that looks smooth, but is nowhere differentiable. The gradient calculated by the standard variational approach correctly describes the topology of this cost function but is of very little help in finding the absolute minimum. Being almost vertical, the gradient will only lead to the neighboring minimum. Sensitivities calculated with the adjoint method give no information on the dependence of the mean position on the parameter. In contrast to this, finite parameter perturbations as applied by investigating macroscopic sensitivity approximately may provide an estimation for the correct dependency. In order to calculated this kind of sensitivity with an adjoint method, Lea et al. (2000) employ an ensemble average of cost function gradients of short time slices of one long integration period to filter the effect of secondary 

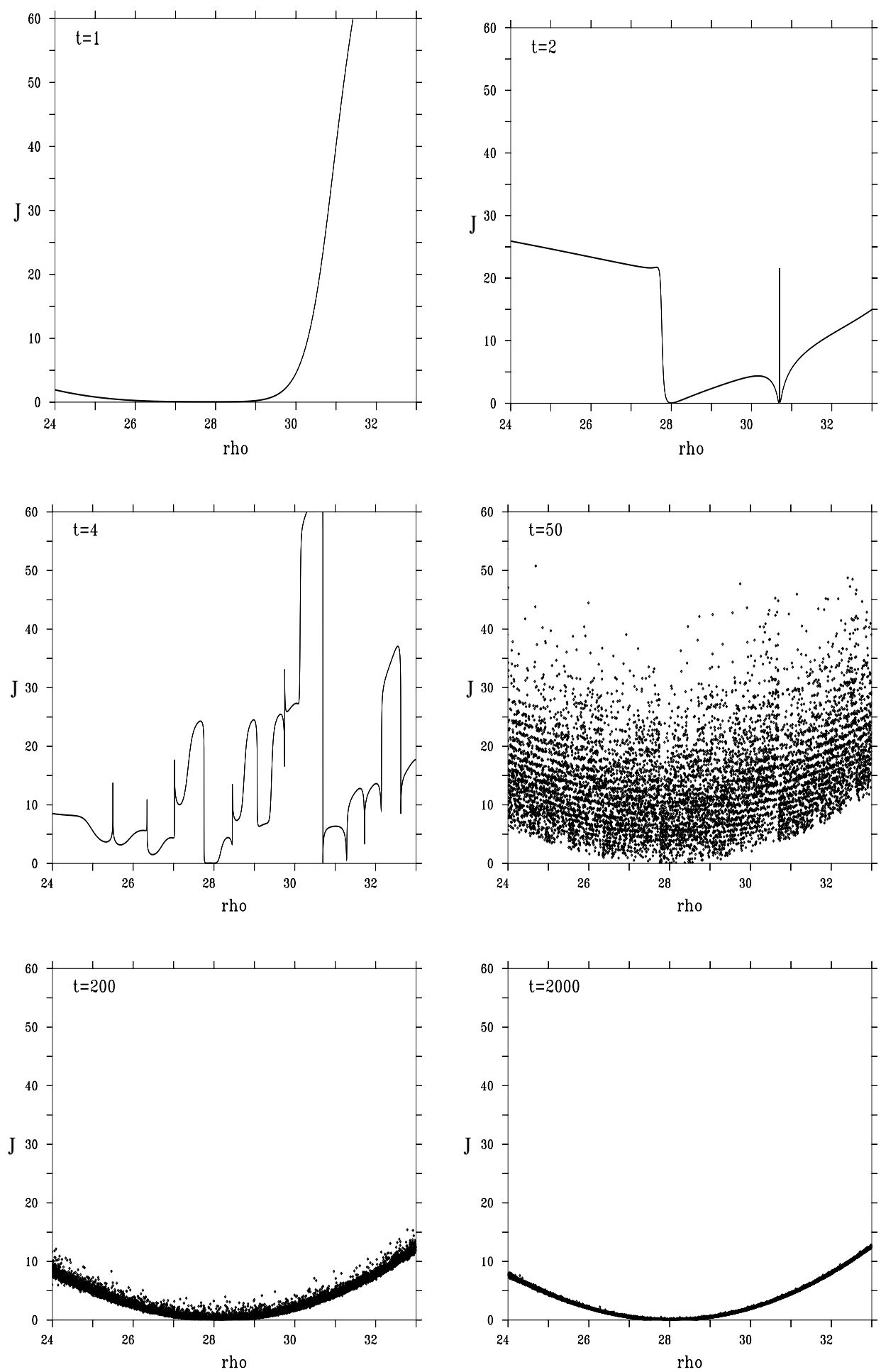

Fig. 2. Evolution of the cost function (4) from the Lorenz model in dependence on the Rayleigh number $\rho$ when increasing the integration period $t$. The transition from a continuous curve into isolated points for $t>50$ is only due to a limited number of cost function evaluations $\left(\Delta \rho=10^{-3}\right)$. The number of secondary minima has then exceeded the number of evaluations. 

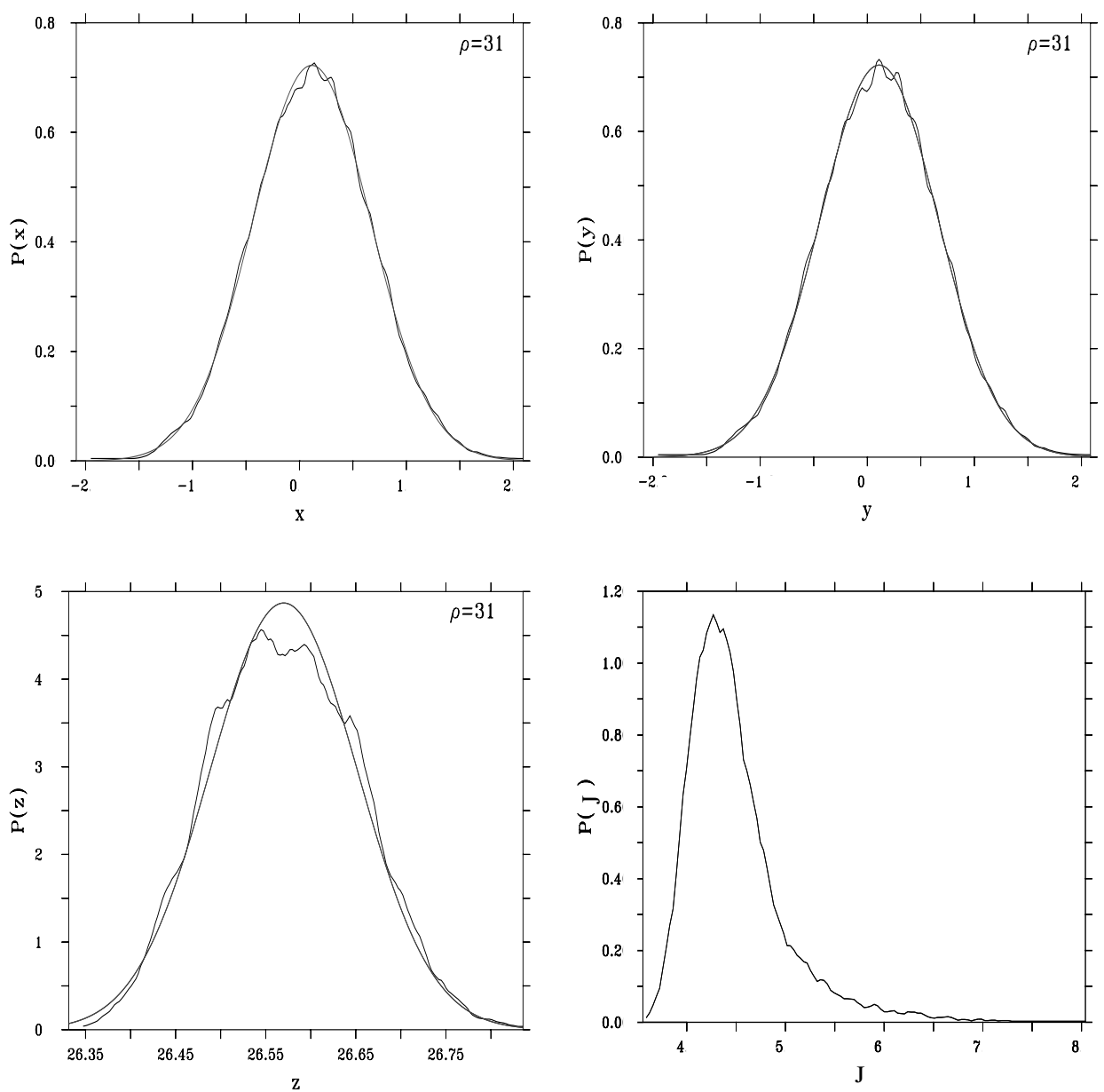

Fig. 3. Probability density functions describing the distribution of the mean values $\bar{x}, \bar{y}$, and $\bar{z}$ and the cost function $J$ of the Lorenz model together with Gaussian approximations. The ensemble of realizations is constructed by varying $\rho$ slightly around a mean value of $\rho=31$.

minima. They were able to estimate the correct macroscopic sensitivity for an intermediate window time scale of $t=0.44$. Is is not clear how this approach performs in more complex models where advection is important.

The problem of using the adjoint method in chaotic systems results from its necessity to find a special trajectory, which is possible only within the limits of predictability. Since the adjoint method relies on the ability to control the model output by tuning the parameters, the method must be based on prognostic equations for quantities which are predictable up to the maximum integration time. The flow beyond the predictability limit is not controllable by parameter changes. In the case of the Lorenz model an independent model capable of predicting an expectation values for $\bar{x}$, $\bar{y}$ and $\bar{z}$ would be a possible basis. It would describe a curve that fits the maximum of a PDF that is given by the distribution of the cost function values in Fig. 2. This approach would be able to calculate a 'mean' gradient in the sense that this gradient disregards deviations of the limit curve of Fig. 2. A general approach for a solution is to base the method on prognostic equations for statistical moments that describe the mean values independently of the very sensitive special realizations of the trajectory. This assumes that these 
equations describe a statistically steady system, as in the Lorenz case, or have at least an inherently longer predictability time scale. A formulation of a method based on this idea that also keeps the advantages of a high-resolution forward model is described in the following section. As one referee noted, cost functions based on statistical moments may show multi-valued characteristics, meaning that for a small parameter range cost function values may accumulate near two or more different curves. The independent description of the statistical moments can not capture this kind of behavior and the method will thus be formulated under the prerequisite of a single-valued cost function.

\section{Method}

The need for a separate adjoint to a model that describes PDFs or mean values independently of special realizations of a trajectory of the forward model was illustrated in the previous section with the Lorenz model. Equations for the evolution of the PDFs can be easily derived from the model equations when stochastic forcing is added and are given by the Fokker-Planck equation (Risken, 1984). This approach has been used recently to circumvent limitations in strongly nonlinear systems by defining sequential assimilation procedures in terms of PDFs. Miller et al. (1994) and Evensen (1994) derived a generalization of the EKF based on Monte-Carlo estimates of covariance statistics. This method is found to work well in QG models by Evensen and van Leeuwen (1996). It can be regarded as a second-order moment approximation of the more general method of Miller et al. (1999), who applied the Bayes theorem directly to PDFs calculated from the Fokker-Planck equations. Although all necessary statistics could be calculated from the forward model similar to Evensen and van Leeuwen (1996), a possible algorithm for the parameter estimation problem would still require the integration of an adjoint to the Fokker-Planck equation. This direct method would be impracticable in systems of dimensions higher than three. For the Lorenz model the integration of the Fokker-Planck equations and therefore their adjoint are yet similar in complexity as the integration of the Navier-Stokes equations. A simpler approach has to be considered in the following, since the Fokker-Planck equation of, for example, a simple QG model describes the evolution of PDFs in a space of thousands of dimensions.

Since only statistical moments are intended to be included in the cost function, it is sufficient to regard for the constraint only equations that describe the evolution of moments as they can be obtained by Reynolds decomposition. From the nonlinearity of the evolution equation a closure problem arises, and it becomes necessary to express second-order moments of the state vector in terms of first-order moments. The equation for the evolution of the first-order moment $\hat{x}$ of a general state vector $x$ (here we introduce a new definition of $x$ different to that in the previous section) might symbolically be represented as

$\frac{\mathrm{d} \hat{x}}{\mathrm{~d} t}=\hat{f}(\hat{x})$.

The statistical model in the strong constraint would determine two equations, the forward and an adjoint to the statistical model. However, there is no such model available to calculate the evolution of moments with the required accuracy. Different approximations are therefore applied for the two different models. The scheme in Fig. 4 illustrates the approximations that are described in the following. In the forward part of the adjoint method (left branch) the statistical model (6) is replaced by time averaging the solution of the

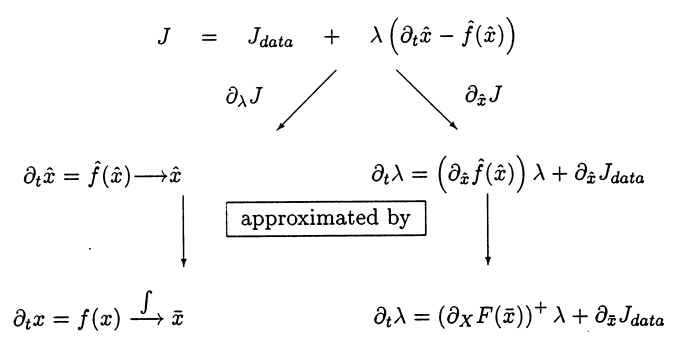

Fig. 4. Outline of the approximation involved in the derivation of the method. The model for statistical moments $\partial_{t} \hat{x}=\hat{f}(\hat{x})$, representing the constraint in the Lagrangian $J$, is approximated in two different ways. Forward part (left branch): The calculation of statistical moments $\hat{x}$ is approximated by averaging the solution $x$ of the high resolution model $\partial_{t} x=f(x)$. Adjoint part (right branch): The adjoint is approximated by the adjoint of a coarse resolution twin model $\partial_{t} X=F(X)$ linearized around the mean value $\bar{x}$ of the high resolution model. 
high-resolution model

$\frac{\mathrm{d} x}{\mathrm{~d} t}=f(x)$.

This is a necessary replacement since state-of-theart realizations $\bar{x}$ of any moment $\hat{x}$ are only available by time-averaging the solution of the eddy-resolving model. A calculation of the solution of the statistical model (6) is not needed. This enables strong approximations for statistical model, since it is only needed for the construction of the adjoint (right branch of the scheme). Being only a tool to calculate the gradient that is used for the optimization, an approximation can still be effective. The standard method is followed and the closure problem is handled by introducing a model twin on a coarser grid,

$\frac{\mathrm{d} X}{\mathrm{~d} t}=F(X)$,

with an increased eddy viscosity that mimics transient processes with short-term predictability. This kind of parameterization usually has shortcomings in regions where nonlinear processes are dominant (see also Fig. 5) but might nevertheless be sufficient to approximate (6) as a basis for the construction of the adjoint model. Realizing that only tangent linear equations are needed in the variational formalism enables a further improvement. In order to construct the adjoint, the coarse resolution model (8) now serving as a simplified model for the moments is expanded at the spatially averaged mean value $\langle\bar{x}\rangle$, which is the best available approximation of the moment. Spatial averages are indicated by \langle\rangle and $\langle\bar{x}\rangle$ describes a realization of a moment calculated from the high-resolution model, as opposed to $\hat{x}$ that derives from the solution of a statistical model.

Courtier et al. (1994) introduced a similar approach with their incremental formulation of the variational assimilation to reduce the cost of the method for an operational implementation. They expand the forward model at the first-guess solution and use a simplification of the first-order tangent linear term for the optimization steps afterwards, and found that the algorithm works well.

Introducing a parameter $\alpha$ of the high-resolution forward model mimicking the controls that are to be estimated, the adjoint operator of the coarse resolution twin model, $\partial_{X} F(X,\langle\alpha\rangle)^{\dagger}$, $\mathbf{O}$ the error covariance of the observations $y, B$ the error covariance of the a priori information, $\alpha_{b}$, of the parameter $\alpha$ and $\mathbf{H}$ the observation operator, the scheme of the assimilation algorithm then reads:

Minimize

$$
\begin{aligned}
J(\alpha)= & \frac{1}{2}\left(\alpha-\alpha_{\mathrm{b}}\right)^{T} \mathbf{B}^{-1}\left(\alpha-\alpha_{\mathrm{b}}\right) \\
& +\frac{1}{2}(\mathbf{H} \bar{x}-y)^{T} \mathbf{O}^{-1}(\mathbf{H} \bar{x}-y)
\end{aligned}
$$

employing the high-resolution forward model (7) to calculate $\bar{x}$ and the adjoint equations

$$
-\frac{\mathrm{d} \lambda}{\mathrm{d} t}=\partial_{X} F(\langle\bar{x}\rangle,\langle\alpha\rangle)^{\dagger} \lambda+\mathbf{H}^{T} \mathbf{O}^{-1}(\mathbf{H}\langle\bar{x}\rangle-\langle y\rangle)
$$

of the coarse-resolution twin (8) to estimate the gradient. The time mean $\bar{x}$ is calculated from the solution of Eq. (7). After spatial averaging to the coarse grid the moment $\langle\bar{x}\rangle$ is inserted into the adjoint equation (10).

This scheme allows the adjoint variables to be propagated on the mean stream lines of the forward model. The transient part, e.g. the eddy-flux terms, are only represented by simple parameterizations. Since the adjoint depends on a model for time-averaged quantities and is defined on a coarse grid, only a subspace of the gradient can be calculated from the adjoint variables and only the part of the parameters that projects onto this subspace can be estimated. This requires either a decomposition of the parameter space to extract the coarse-resolution time-averaged subspace or an interpolation of the gradient.

Approximating the statistical model by a lowresolution model will cause the adjoint variable and therefore the cost function gradients to be only approximate. Further errors are introduced by interpolation and averaging steps which link the high-resolution forward and the low-resolution backward model. However, as in the outer loops in the application of the incremental method by Rabier et al. (1998), no approximations are made within the cost function and the forward calculation. Since the gradient is only a means for finding the minimum, errors in the adjoint variables will possibly reduce the performance with an eventual failure in convergence of the method. This is especially true for the estimation of viscosity coefficients, since the effect of mixing is differently represented in the forward and the model that is used for the construction of the adjoint. Although 
it is formally still possible to calculate gradients with respect to viscosity coefficients, the gradient may not be helpful at all. However, the shape of the cost function and the position of the minimum will not be affected in any case. Sensitivities calculated by this method are very likely to be subject to a bias, especially concerning the amplitude and exact positions of spatial features.

The error covariance $\mathbf{O}$ is a function of the measurement errors but also incorporates the limited representativeness of the observations due to variability of the physical system. Averaged quantities appear in high-resolution models as stochastic quantities. An estimation of the covariance $\mathbf{O}$ is possible from an ensemble of model integrations, assuming that the modeled variance of the statistical moments is a good approximation of the variance of moments of real data and that the measurement errors are negligible in comparison. Systematic errors are not yet taken into account. Presuming uncorrelated errors, information about a systematic bias can combined with the ensemble error estimate afterwards.

The method is so far only applicable for mean values. The extension of this method for the assimilation of higher-order moments is not straightforward. A simple approach that uses parameterization schemes is presented in Section 4.4.

\section{Assimilation experiments}

To evaluate the performance of the method, experiments are performed with a QG model. The new method will be first tested in an identical twin configuration where the perfect model assumption holds and results can be tested against fields and parameters of a reference experiment. The general strategy for all experiments is to reconstruct the zonal wind stress curl from simulated statistical moments such as mean stream function and stream function variance expressed as sea surface height (SSH) variance. After an integration time of $20 \mathrm{yr}$ from a state of rest, the model approximately is in a statistically stationary state.

\subsection{Model description}

The three-layer QG model is based on the Holland (1978) model and basically identical to that described in Vogeler and Schröter (1995). A limited area double gyre configuration is set up on the $\beta$-plane with the Coriolis parameter at a central latitude of $40^{\circ}$ to mimic a simple model for mid-latitude jets. The resolution is $1 / 6^{\circ}$ in the zonal and meridional direction and the area extends meridionally from $32^{\circ} \mathrm{N}$ to $48^{\circ} \mathrm{N}$. The layer thicknesses are from top to bottom 300,700 and $4000 \mathrm{~m}$, respectively, and reduced gravities at the layer interfaces are 0.0357 and $0.0162 \mathrm{~m} \mathrm{~s}^{-2}$. Friction coefficients are $10^{-7} \mathrm{~s}^{-1}$ for the bottom friction and $10^{2} \mathrm{~m}^{2} \mathrm{~s}^{-1}$ for harmonic lateral friction. The reference experiment is forced with zonal wind stress given by $\tau=\tau_{0} \cos \left(2 \pi y / L_{y}\right)$ with $\tau_{0}=$ $10^{-4} \mathrm{~m}^{2} \mathrm{~s}^{-2}$. A flat bottom is prescribed, and free slip conditions are applied at the closed boundaries. The mean upper layer stream function (Fig. 5) is a statistical stationary double gyre. The maximum zonal velocity is about $1 \mathrm{~m} \mathrm{~s}^{-1}$ and the maximum r.m.s. SSH variability about $60 \mathrm{~cm}$.

\subsection{Adjoint model}

The prognostic model used for the construction of the adjoint for stream function moments is identical to that described above except of a decrease in resolution to $2 / 3^{\circ}$ and an increase of the lateral friction to $10^{4} \mathrm{~m}^{2} \mathrm{~s}^{-1}$. The solution of this configuration is a stationary two-gyre system with a reduced penetration scale and velocity of the zonal jet (Fig. 5b). The adjoint to the QG model is described in Moore (1991) and Schröeter (1993). A quasi-Newton method based on the Davidon-Fletcher algorithm is chosen to minimize the cost function. The algorithm was successfully applied by Jung et al. (1998). The discretization in time is changed to a finite difference of the adjoint analogue to Sirkes and Tziperman (1997) in order to suppress the computational mode. Since Eqs. (10) are linear autonomous equations for calculation of the adjoint variable $\lambda$, the system represents a simple method, the relaxation method, for the calculation of the stationary solution. The adjoint step, therefore, is accelerated using a Gauss-Seidel solver, a more efficient scheme. Calculating the stationary solution is found to perform more efficiently in the optimization and is used throughout the following experiments. In order to avoid a decomposition of the parameter space into the coarse resolution portion and its fine-scale complement, the simpler approach is taken and the gradient is interpolated 
a

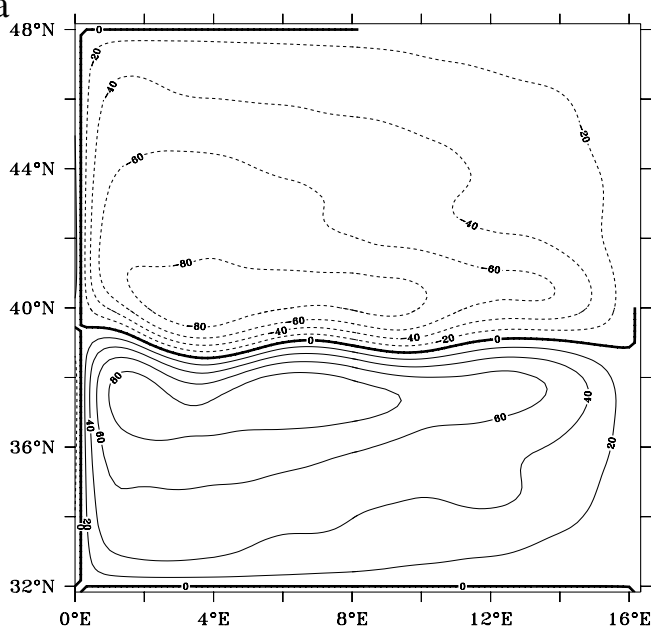

$\mathrm{b}$

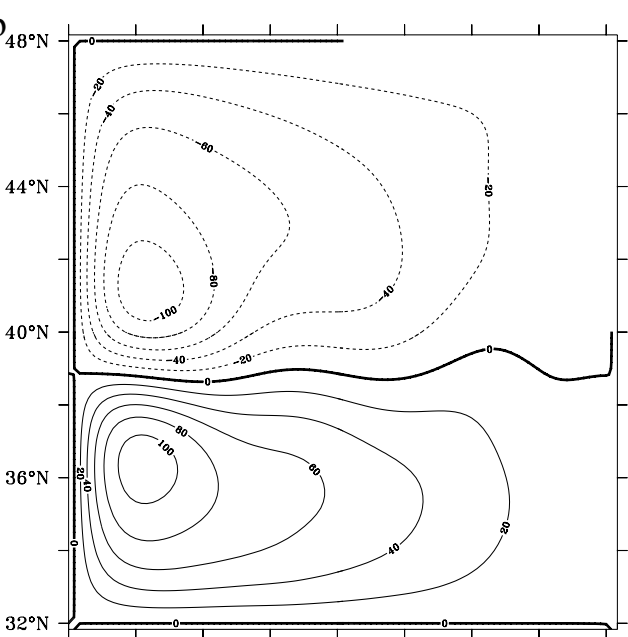

Fig. 5. Annual mean upper layer stream function (in Sv) from the high-resolution experiment (a) and a coarseresolution experiment (b). Both experiments are taken from the 21 th year of integration with identical wind forcing as shown in Fig. 12. The coarse-resolution model is the $2 / 3^{\circ}$ twin from which the adjoint was constructed and therefore identical to the high-resolution model at $1 / 6^{\circ}$ except for resolution and horizontal mixing. The highresolution run is used as reference experiment from which simulated data are taken.

to the high-resolution grid. The descent step is then applied within parameter space of the forward model.

\subsection{Assimilation of stream function data}

To measure the least-squares distance between the actual upper layer mean stream function $\overline{\psi_{1}}$ and the simulated observations from a model run with reference wind forcing, a quadratic cost function

$J(\tau)=\frac{1}{N} \sum_{i} \frac{\left[\overline{\psi_{i, 1}}(\tau)-\overline{\psi_{i, 1}}\left(\tau_{0}\right)\right]^{2}}{\varepsilon^{2}}$

is introduced where the summation index $i$ is a spatial index denoting the horizontal position. All experiments are started from the same initial condition, which is the state after $20 \mathrm{yr}$ of forward integration with reference wind forcing. Mean values were derived from an integration period of $1 \mathrm{yr}$. The control parameter is the curl of the zonal wind stress vector $\tau$.

The error covariance matrix $\mathbf{O}$ is assumed to be diagonal and spatially homogeneous. Therefore, the variance $\varepsilon^{2}$ is the spatial average of the variance of an ensemble of mean stream functions, e.g. the diagonal variance of $\mathbf{O}$. The ensemble members are derived from varying the reference wind stress curl by an amount of less than $5 \%$. Due to this choice of $\varepsilon^{2}$ the cost function shows values of about one in the vicinity of the minimum. Since the moments of high-resolution models are regarded as stochastic quantities no additional noise is added to the simulated observation.

4.3.1. Behavior of the cost function. For a comparison with the results from the Lorenz model, a section through the cost function is made. The wind stress patterns that correspond to this section are obtained by decomposing the reference function into discrete wavelet modes (Press et al., 1993) and tuning the amplitude of the fourth mode, which causes mainly large-scale variations of the wind stress. A decomposition into wavelet modes is chosen to allow for an easy reduction of the number of degrees of freedom, realized in an experiment described below. It was shown by Farge (1992), who also gives a review about wavelets, that data from turbulence may be reconstructed fairly well by using only a limited number of wavelet modes. The modes depend on a scale and a position parameter. The first two modes describe the mean value of the function. The 
remainder of the space is classified by a sequence of $N$ sets, $n=1 \ldots N$. Each set contains $2^{n}$ modes of an identical wavelength proportional to $2^{-n}$, starting for $n=1$ with the wavelength that corresponds to the length of the whole interval. The reference wind stress is thus described essentially by the third and fourth mode. The reference wind stress and the pattern resulting from doubling the fourth coefficient is shown later in Fig. 12.

Figure 6 shows the section through the cost function. The curve shows some analogies to the corresponding curve for the Lorenz system (Fig. 2) and resembles one with stochastic deviations superposed on a parabolic shape. The smooth black curve in Fig. 6 describes the cost function that results from a simulation with the low resolution $2 / 3^{\circ}$ model on which the adjoint is based. The large displacement of this curve indicates that no consistent solution can be found with that

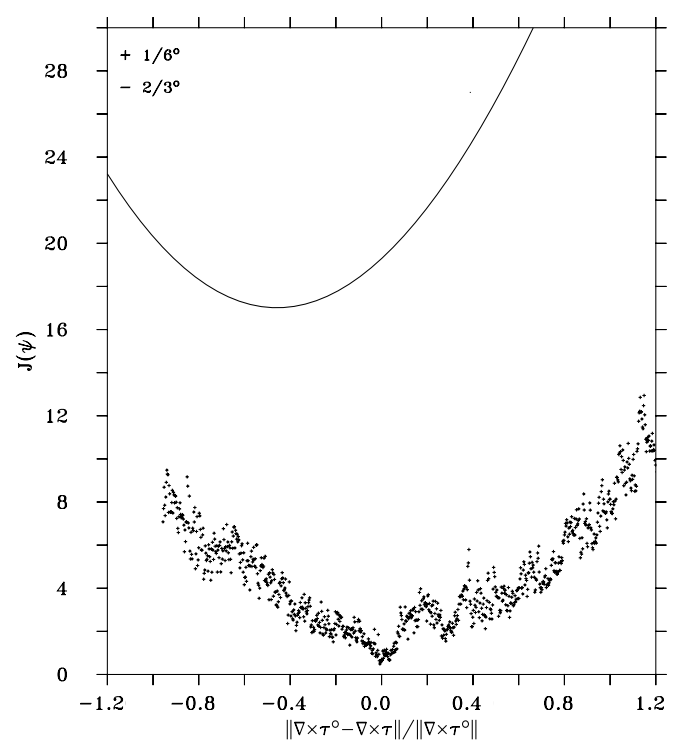

Fig. 6. Samples from sections through cost functions based on annual mean upper layer stream functions. Simulated observations are from the high-resolution model with the reference wind forcing $\tau^{\circ}$. The dependence on the parameter $\nabla \times \tau$, which is effectively the fourth mode of a wavelet decomposition, is displayed by the normalized Euclidean difference to $\nabla \times \tau^{\circ}$. The crosses correspond to experiments with the high-resolution $1 / 6^{\circ}$ forward model. The smooth curve is the cost function that results from simulations with the low-resolution $2 / 3^{\circ}$ model. model. The shift in the minimum shows that the parameters estimated from an optimization with that model are significantly in error, due to systematic model errors. However, the resemblance of the shape of the cost function to the high-resolution analogue suggests that a linearization approach about the mean calculated from the solution of the eddy resolving model may work fairly well. The precision up to which a parameter can be retrieved is expected to depend on the noise level and the curvature of the cost function. A criterion for terminating the optimization is given by a reduction below the noise level, e.g. a cost function value of one. This is not enforced during most of the following experiments to investigate the order of magnitude of the possible reduction.

4.3.2. Assimilation period of $1 \mathrm{yr}$. In this section zonal wind stress curl is estimated from annual mean stream function data. A period of $1 \mathrm{yr}$ is not sufficient for the model to equilibrate to changes in wind stress. The reference experiment from which the data are taken and the assimilation experiment are started from identical initial conditions (only the wind stress is changed). The wind stress is thence estimated from the response of the stream function to the changed wind stress instead of estimating wind stress from a quasi-stationary state. This experiment indicates whether the response of statistical moments (the annual mean upper layer stream function) to a parameter change shows enhanced predictability in comparison to the time scale that is associated with the inherent chaotic dynamics at the mesoscale.

The optimization, in this and all subsequent experiments, is started from a control parameter that is obtained by changing the fourth mode of the reference wind stress. Paths from minimizing the cost function (11) by estimating the zonal wind stress are shown in Fig. 7 against the normalized Euclidean distance to the true wind stress curl. The paths generally leave the line that is marked by the section through the cost function, since the optimization space is not one-dimensional. Cost function values therefore may show considerably higher or lower values for the same Euclidean distance, even if the cost function would be free of noise. The optimization is assessed in the figure simultaneously on basis of the distance in parameter and observation space. 


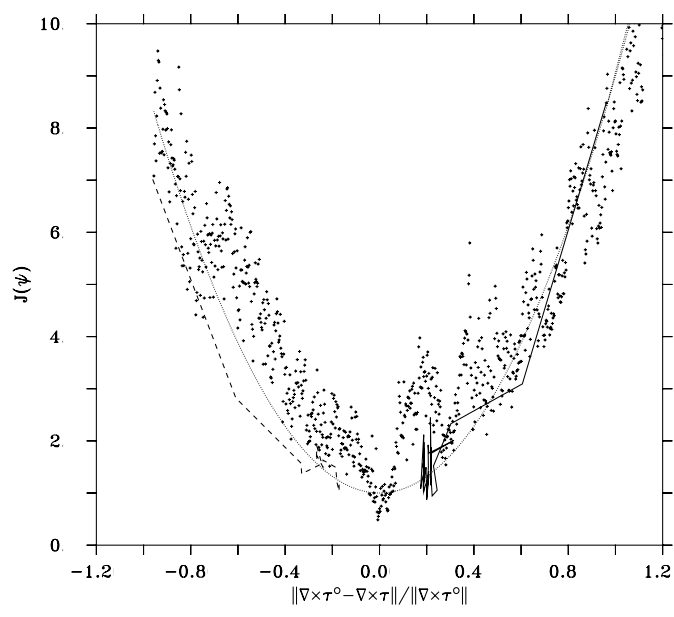

Fig. 7. Samples from a section through the cost function from Fig. 6 together with paths from optimizing the zonal wind stress $\tau$. The cost-function values are based on annual mean upper-layer stream functions. The parameter of the optimization is the full zonal wind stress Both sets are displayed as a function of the Euclidean distance to the reference wind stress $\tau^{\circ}$. The solid path is from an experiment that employs one realization. An ensemble of 10 realizations is used for the dashed path shown on the left-hand side for clarity. Ensemble members are derived by varying the wind stress slightly by less than $5 \%$. The cost-function values are then computed as a mean over the realizations. The parabola is fitted by hand and used for an a posteriori error estimate.

For the dashed path in Fig. 7 an ensemble of 10 realizations was used to investigate whether a reduction of the noise level results in a higher precision of the parameter estimation. The members are constructed by varying slightly the parameter by less than $5 \%$. The ensemble was used to construct an ensemble mean stream and cost function. However, a single adjoint that is obtained from linearizing around the ensemble mean is employed to calculate cost function gradients. The effect is similar to extending the period of assimilation in terms of reducing eddy effects. The resulting ensemble variance reflects the spurious eddy effect on the annual mean stream function which was already used to define $\varepsilon^{2}$. The expected effect on the precision is not captured, and the final state is virtually of the same quality as in the experiment on the basis of one single realization. The Euclidean distance between the two final parameters is of the same order as the distance to the reference value.
A parameter null-space that is unobservable from mean stream function data may account for the remaining difference from the reference parameter and the failure in improving the estimation by ensemble information. This is investigated by performing the optimization in wavelet space instead of physical space and by restricting the parameter space to the four first modes of the wavelet decomposition. Since this optimization ends up with the same distance to the minimum (not shown), it is not likely that a null-space accounts for the limitation. The precisions is either be limited by a general error of the gradient due to the approximation necessary within this method or by trapping into larger secondary minima (such as the one visible in Fig. 7) that were not ignored by the calculation of the gradient with the coarse resolution adjoint.

The important part in estimating the posterior error covariance of parameters is according to Thacker (1989) the inverse of the Hessian matrix of the cost function. An approximation to the inverse of the Hessian is being constructed by the quasi-Newton algorithm. The calculation of the Hessian requires about 100 adjoint computations for the present problem of estimating the zonal wind stress curl which still would be feasible. However, for our method it is expected that at the final stage the estimation of the Hessian is corrupted by the variability of the gradient, which is then strongly subject to the stochastic nature of the cost function. However, linear Sverdrup theory predicts a direct relation between zonal wind stress and stream function. It seems thus sensible to assume isotropy for the dependence of the cost function on the parameter, which means that the cost function is expected to depend mainly on the Euclidean distance of the wind stress to the reference value. This is supported by the optimization experiments, since the paths from all optimizations trace the shape of the one section through the cost function. A simple approach is then to estimate the Hessian by fitting the shape of the cost function in Fig. 7 to a parabola. All parabolas drawn together with sections through cost functions are obtained by adjusting the curve by hand under the constraint that the value in the minimum is one accounting for the normalization of the cost function with the error variance. By this way, a relative error of $25 \%$ of the zonal wind stress curl is estimated from this approximation of the 
Hessian which is in correspondence with the achieved distance of the parameter estimation.

4.3.3. Assimilation period of $5 \mathrm{yr}$. A period of $5 \mathrm{yr}$ is chosen to precede the assimilation to allow for equilibration to the changed wind forcing, and the period of assimilation is extended to a further $5 \mathrm{yr}$ from which the stream functions are calculated to reduce stochastic-like deviations caused by different eddy realizations. A period of $5 \mathrm{yr}$ derives from the response time of the model to changed wind stress, which is a little less than $5 \mathrm{yr}$. In order to substantially reduce the cost function noise related to low-frequency oscillation on a decadal time scale Meacham (2000), much larger integration periods are necessary but not feasible. The definition of the cost function and the estimation of the error covariance is analogous to Section 4.3.2.

The amplitude of the stochastic-like component of the cost function shown in Fig. 8 is noticeably increased in comparison to the 1 -yr experiment. Though eddy effects on the mean stream function are decreased similar to the ensemble experiment

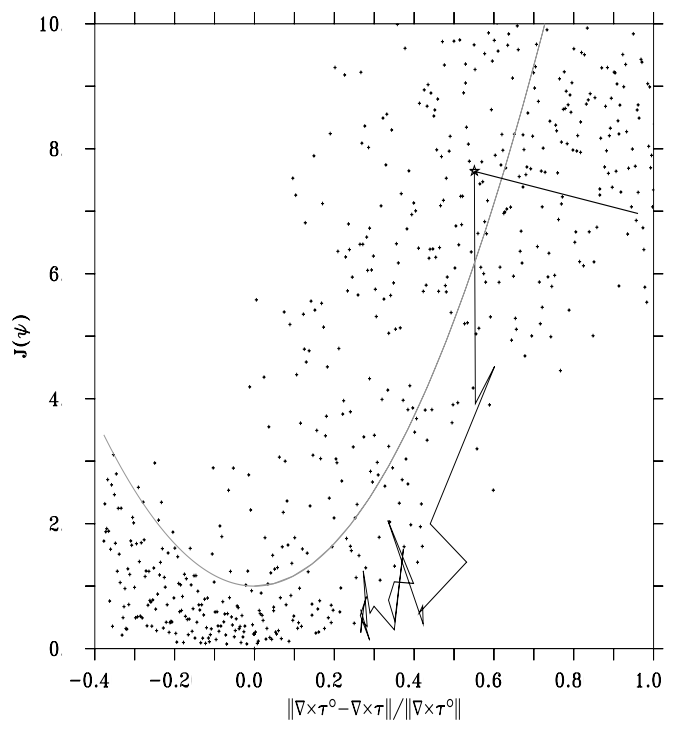

Fig. 8. Samples from a section through the cost function based on mean upper layer stream functions derived from an integration period of $5 \mathrm{yr}$ together with a path from optimizing the curl of the zonal wind stress $\tau$. The star denotes a restart of the descent algorithm. The parabola is fitted to the cost function by hand of the last section, a second source of variability influences the cost function. The QG model involves a wider spectrum of different time scales than the Lorenz model. The cost function values are, in contrast to the 1-yr integration, now subjected to low-frequency variability of the jet stream position (Meacham, 2000). Very small cost function values thus exist even for large parameter errors which makes parameter estimations difficult.

Cost function values can increase even if parameter values are moved closer to truth if a parameter realization in the upper range of the cost function variability occurs. Therefore small improvement steps are generally unfavorable. They will hinder the minimization process, because the gradient does not contain information about the fine structure of the cost function. Usually this is only a problem if it happens during the first steps, where the Hessian is only insufficiently determined by prior steps. The optimization was therefore restarted after the first step. For an application with real data it is, however, not possible to know whether the first parameter change in fact leads to a parameter improvement. An unfavorable choice of the step size may deteriorate the parameter estimate, and a restart would lead further away from the optimal value. The value of the step sizes was thus tuned before in coarse resolution identical twin experiments. With the assumption of a favorable parameter change, a restart is then suggested. Another possibility is the ensemble approach that provides an estimation for the variability of the cost function values and information about the location of a realization with respect to the ensemble mean.

The path from the optimization in Fig. 8 shows that a final cost function value is found well below the noise level in the vicinity of the minimum. All cost function evaluations are plotted in the figure. All cost function values of the last 10 iterations are below the noise level, indicating that despite the large relative wind stress curl error of about $30 \%$, a wind stress vector is found that causes the mean stream function to be in reasonable agreement with the assimilated data. In terms of cost function values, the estimated parameter is thus of the same quality as one that is much closer to the reference parameter. The final parameter value has a normalized Euclidean distance to the correct parameter of about $27 \%$. The error estimation 
gives a relative error of $17 \%$ which is lower than the $1 \mathrm{yr}$ averaging case, because the error is only determined by the curvature of the parabola and the noise level in the vicinity of the minimum which was higher for the 1-yr period in comparison to the 5-yr experiment. Most of the points on the path of the optimization are significantly below the cloud of samples from the cost function, showing that the assumption that the cost function mainly depends on the Euclidean distance of the wind stress to the reference only holds marginally.

The 1-yr experiments show a better performance than the 5-yr experiment in terms of relocating the wind stress, but are less able to reproduce the mean stream function that is assimilated. This is not surprising, since for the 5-yr experiment samples with very low cost function values are densely distributed in the range of a $20 \%$ wind stress curl error, whereas values below 0.5 are not found in the 1-yr cost function in Fig. 7. The success of the 1-yr experiment relies on the correct initial condition. The 5-yr adjustment period that precedes the 5-yr experiment decouples the stream function from the influence of the initial condition. In the former experiment wind stress is thus estimated from the response of the stream function to the changed wind stress but estimated from a quasi stationary stream function in the latter.

\subsection{Assimilation of variance data}

The assimilation of higher-order moments is especially interesting in context with the use of altimeter data. Due to the lack of a precise geoid, only anomalies of the sea surface elevation are in general assimilated in circulation models. The sequential assimilation method of Oschlies and Willebrand (1996) projects surface velocity increments onto deep velocity increments and calculates the corresponding hydrographic adjustments from an inversion of the thermal wind equation. An application shows that although variability was markedly improved, the mean position of the front which is associated with the variability was not corrected. However, the strong dynamical link between frontal structures and the corresponding variability enables in principal the correction of the mean frontal position (which is related to a first-order moment) by assimilating only information from second order moments as they are available from $\mathrm{SSH}$ variance.
Sophisticated closure schemes and models for higher-order moments are, however, only available for idealized geometries and forcing functions, e.g. Holloway and Hendershott (1977), and would be computationally impracticable. In this section simple schemes for parameterizing the SSH variance in the adjoint equations are considered for the assimilation of $\mathrm{SSH}$ variance.

A large fraction of eddy variability in ocean and atmosphere models is generated due to instability processes that transfer energy from the mean velocity to the transient part. Typical mechanisms are baroclinic and barotropic instability arising from vertical and horizontal shear of the mean velocity. In order to avoid contamination by noise from differentiation, the mean upper layer velocity is used instead of velocity shear to parameterize variability:

$\sigma^{2}=\kappa^{2}|\bar{u}|^{2}=\kappa^{2}\left(\left[\partial_{x} \bar{\psi}_{1}\right]^{2}+\left[\partial_{y} \bar{\psi}_{1}\right]^{2}\right)$.

The mean is the temporal mean only and $\kappa$ is an empirical coefficient of proportionality. The mean horizontal and vertical shear can be approximated by the mean velocity divided by a typical horizontal and vertical scale of the zonal jet, respectively. Horizontal and an vertical scales are assumed to be constant which is supported by almost constant width of the zonal jet stream and the small lower layer velocities. The proportionality to the square of the velocity is assumed in analogy to the parameterization of eddy fluxes derived by Green (1970) and Stone (1972) for the baroclinic instability. A similar relation that involves mean kinetic energy derived from thermal wind was used by Stammer (1997) to characterize regions of high eddy kinetic energy.

Figure 9 shows that except for the boundary regions a pattern similar to the variability can be obtained by the parameterization from the mean stream function. Only the relation between variations of SSH variability and velocity variations enter the adjoint equations. In contrast to Eq. (12), an additional additive constant can therefore be included into the estimation of $\kappa$. The coefficient $\kappa \approx 0.3 \mathrm{~s}$ is estimated from the linear regression displayed in Fig. 10. Typical regression coefficients are between $r=0.7$ and $r=0.9$.

The definition of the cost function based on the stream function variance, expressed as $\mathrm{SSH}$ variance $\sigma^{2}$ through the relation $\mathrm{SSH}=f_{0} \psi_{1} / g$, is 

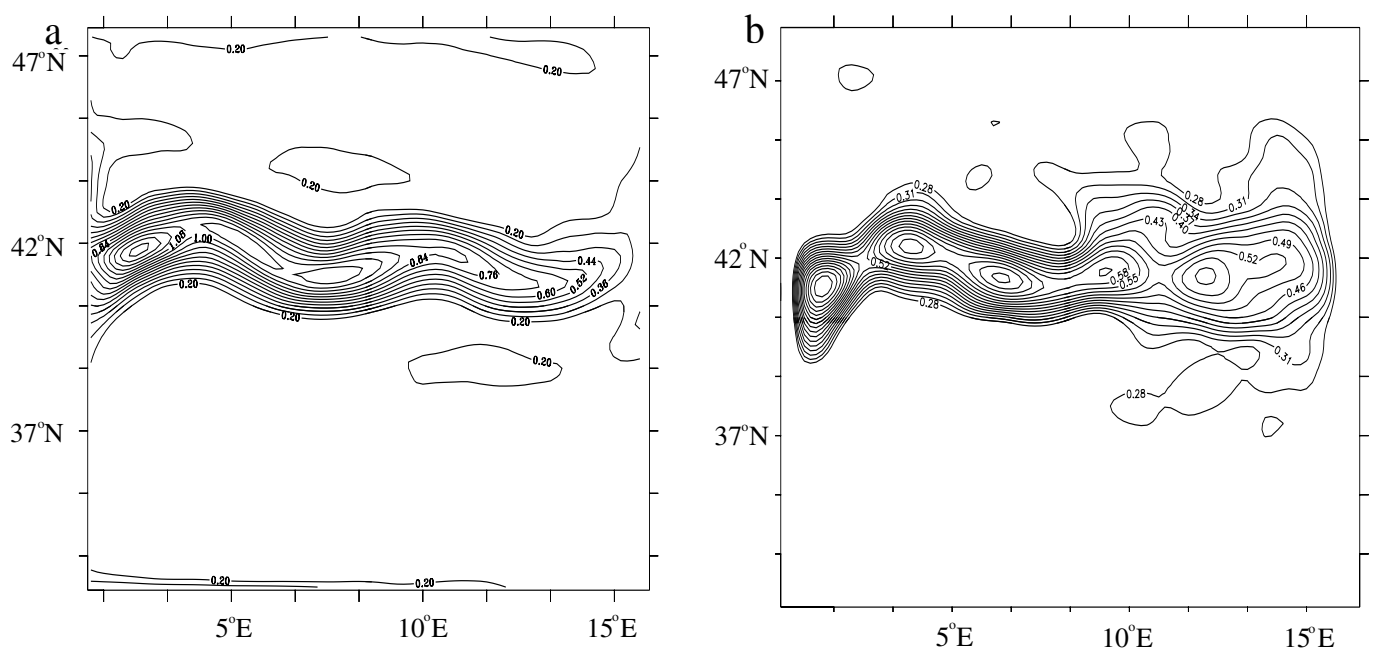

Fig. 9. Mean velocity $\left(a\right.$, in $\left.\mathrm{m} \mathrm{s}^{-1}\right)$ and $\mathrm{SSH}$ variability $(b$, in $\mathrm{m})$, derived from a high-resolution model integration of $2 \mathrm{yr}$.

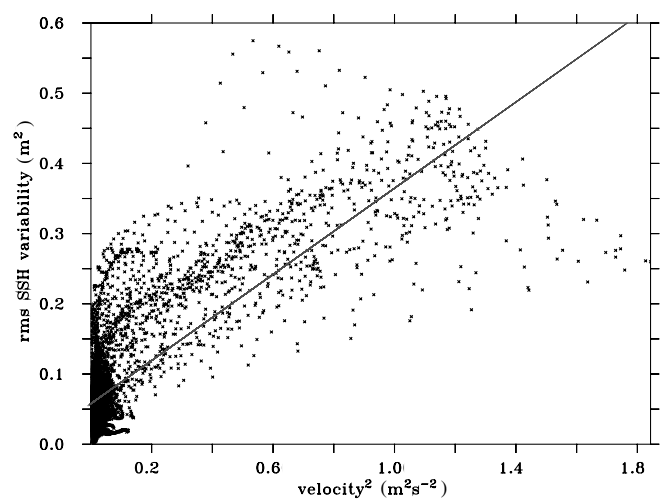

Fig. 10. Regression of the patterns shown in Fig. 9. Five points closest to the boundaries are disregarded to exclude boundary currents which are not related to SSH variability. The correlation coefficient is $r=0.82$.

analogous to Section 4.3:

$J(\tau)=\frac{1}{N} \sum_{i} \frac{\left[\sigma_{i, 1}^{2}(\tau)-\sigma_{i, 1}^{2}\left(\tau_{0}\right)\right]^{2}}{\varepsilon_{\sigma}^{2}}$,

and the same strategy is used for estimating the error $\varepsilon_{\sigma}^{2}$. In contrast to previous experiments concerning mean stream function data, it appears that within a period of $1 \mathrm{yr}$ the stream function changes too little to significantly influence the variance. For this reason the same two periods of $5 \mathrm{yr}$ for assimilation and equilibration as in the last section are chosen.

The amplitude of the stochastic-like component of the cost function based on variance data and illustrated in Fig. 11 is very similar to the analog function based on mean stream functions. The first-guess wind stress is the same as in the previous section (see also Fig. 12). The path in parameter space obtained by optimizing the wind stress shows nearly no convergence to the correct value, although the cost function is reduced to a value below the noise level. The jet is moved from the southward displaced position to a position that approximately corresponds to the observations (not shown). The wind stress curl shown in Fig. 12 reveals that the curve in the vicinity of the line of zero wind stress curl, which in linear Sverdrup theory is the position of the jet, is very well recovered, but shows nearly no convergence elsewhere. In order to examine whether this feature is robust against changing the initial guess for the wind stress a second experiment was performed. The wind stress curl is derived from a point reflection of the wind stress curl of the previous experiment (Fig. 13), thus shifting the position of the jet to the north instead of to the south. The optimization path displayed in Fig. 11 shows the same feature as before and is reduced below the noise level while the parameter is almost not 


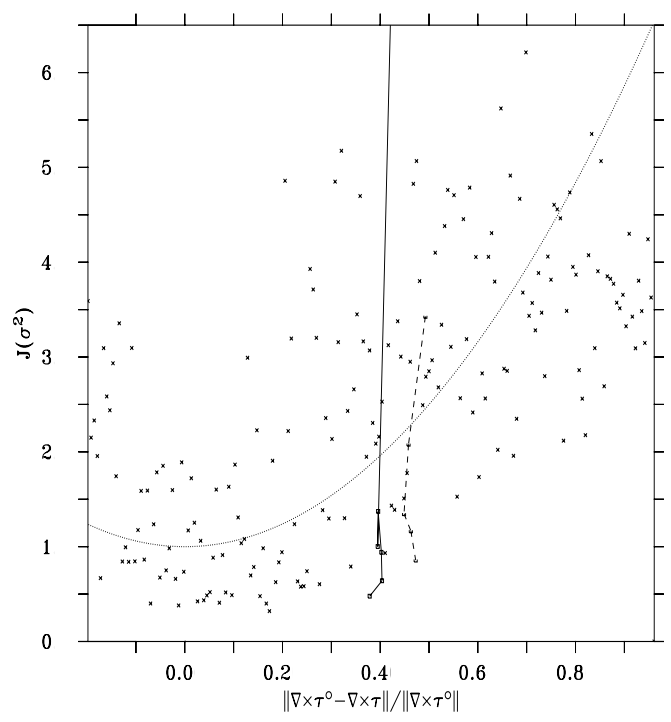

Fig. 11. Samples from a section through the cost function based on SSH variances taken from an integration period of $5 \mathrm{yr}$, together with paths from optimizing the zonal wind stress $\tau$ with the parameterization from Section 4.4. The paths correspond to the first-guess wind stress curl displayed in Fig. 12 (dashed) and Fig. 13 (solid). The first cost-function value of the solid path is 20.2 and out of the region. The parabola is fitted by hand to the samples from the cost function.

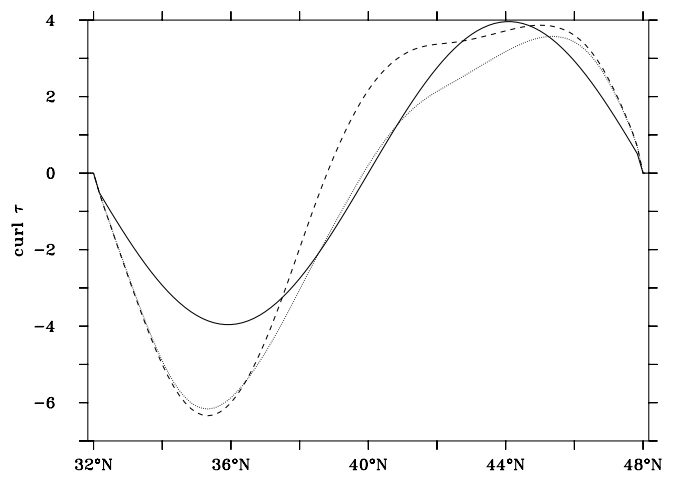

Fig. 12. Curl of the wind stress $\tau$ (in $10^{-4} \mathrm{~m}^{2} \mathrm{~s}^{-2}$ ): reference (solid), first guess obtained by replacing the amplitude of the fourth mode of the wavelet decomposition by twice the value (dashed) and the final iteration (dotted).

improved at all. The position of the zero curl line is almost correct; however, a good fit in the vicinity of the zero curl line is not established. Being almost antisymmetric the wind stress curl still

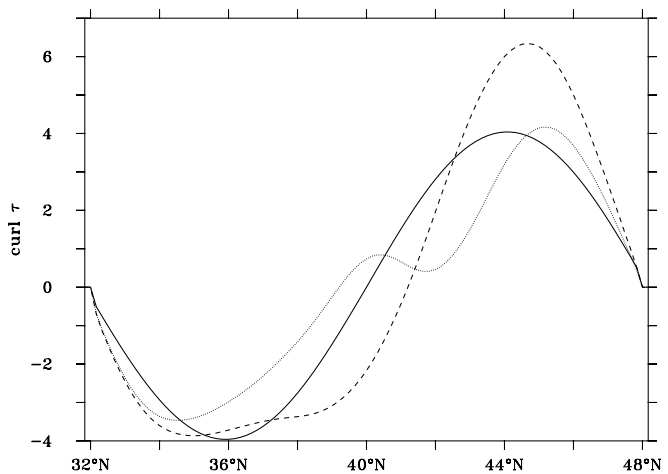

Fig. 13. Curl of the wind stress $\tau$ (in $10^{-4} \mathrm{~m}^{2} \mathrm{~s}^{-2}$ ): the first guess wind stress curl (dashed) derives from point reflection of the first guess of Fig. 12. The solid and the dotted curve correspond to the reference experiment and final iteration, respectively.

leads to jet stream position similar to the one that corresponds to the reference experiment. This is not surprising, since Fig. 9 demonstrates the close relation between the mean position of high variability and the mean current.

Low sensitivities in the in combination with a large noise level of the cost function introduce a unobservable null-space that can not be recovered from SSH variance alone. The experiment employing mean stream function data performed better in relocating the correct wind stress curl. However, it is not clear whether the result is robust with respect to a change of the initial parameter choice. Investigating the relation between the improvement of variance and the improvement of the mean stream function, our actual motivation for the experiment with variance data, clarifies this point.

The first variance assimilation experiment is analyzed by constructing two ensembles from varying slightly the first guess and final parameter, respectively. In Fig. 14 the two ensembles of cost function values based on mean stream function, $J(\bar{\psi})$, are then plotted against the corresponding ensembles defined on basis of variance, $J\left(\sigma^{2}\right)$. The ensemble corresponding to the final parameter shows values around one for both cost function types. The mean stream function and thus the mean frontal position is improved and in accord with the stream function of the reference. Being a promising result for the possibility of relocating mean frontal positions by assimilating variance 


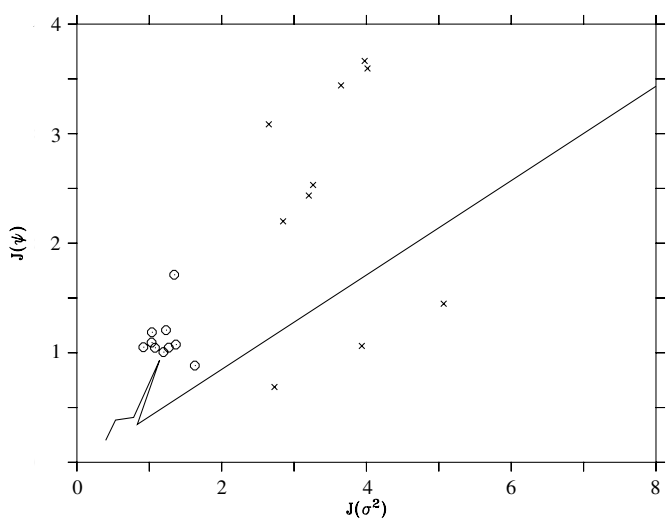

Fig. 14. Cost-function values of the mean stream function $J(\bar{\psi})$ versus the SSH variance cost function $J\left(\sigma^{2}\right)$. Values denoted by $(x)$ and $(\bigcirc)$ are a posteriori ensembles corresponding to the first guess and final wind stress curl of the variance assimilation shown in Fig. 11 (dashed curve experiment). Both ensembles are generated by slightly varying the wind stress by less than $0.1 \%$. The curve shown corresponds to the solid curve of Fig. 11.

data only, the experiment puts the success of the assimilation of stream function into perspective yet. The same implication is supported by the optimization path that is drawn in Fig. 14. The cost function values, $J(\bar{\psi})$, for the final wind stress of both variance assimilation experiments are already consistent with the noise level of the stream function cost function. Starting from this parameter, an assimilation of stream function data would be with little perspective. Low $J(\bar{\psi})$ values for large parameter errors as constructed by assimilating variance data only, demonstrate that the stream function cost function is probably as anisotropic as the variance cost function.

Altogether, the potential for relocating the wind stress is quite low, relocating the mean stream function pattern by assimilating variance data only seems to be better conditioned. An experiment of estimating 2D wind stress curl fields by assimilating mean stream function data (not shown) supports this result. The cost function is reduced below the noise level, while the parameter error of the final estimate of the wind stress not reduced. High sensitivities in the eastern part of the basin led to large parameter changes in this part of the basin whereas the wind stress in the western part remains almost unchanged. Changes of wind stress are not closely enough linked to the circulation pattern changes (meaning that a large subspace of almost equivalent wind stress functions exist) especially because large low-frequency variability of the stream function makes slight changes of the dynamical behavior virtually undetectable. This implies in reverse that the exact wind stress is not inferable from circulation characteristics.

\section{Conclusion}

The experiments presented here demonstrate that it is possible to improve the state of a dynamical system by assimilating statistical moments. We have here derived an extension of the original adjoint formulation which offers a way for improving parameters in high-resolution ocean models with chaotic dynamics. The method may be used to correct systematic errors and in a statistical sense for state estimations with eddy resolving models.

The standard adjoint method is limited by the predictability of the dynamical system. Secondary minima emerge when the period of assimilation is increased beyond the predictability limit and hinder the convergence of the method. Statistical characteristics remain predictable for much longer periods than the eddy time scale and form the basis of the method for increasing the assimilation period. The cost function is thus based on statistical moments and as a prerequisite for the formulation of the method, the eddy-resolving model is used for calculation of the moments. Secondary minima still emerge since only realizations of the moments are calculated from the solution of the high-resolution model. For long integration periods the moments could be regarded as single realizations of Gaussian distributed moments which change smoothly for variations of parameters. This was shown to be true in the case of the Lorenz model.

The new approach applies the adjoint to a prognostic model that describes the dynamics of the moments. The formalism is able to calculate cost function gradients that ignore the fine structure of secondary minima. The application of coarse resolution models as approximations for the moment models makes it necessary to add special parameterization schemes for the assimilation of higher-order moments. Very simple para- 
meterization approaches are tested for this purpose and shown to be able to predict gradients which could be applied for efficient optimization and thus parameter estimation. A limited number of iteration steps are sufficient to minimize below the noise level. The stochastic nature of moments calculated from high-resolution models enables a consistent estimation of error covariances from ensembles of simulations.

Since the model of statistical moments was selected to be only approximate, the choice of this model is in a certain sense arbitrary. As for the construction of ocean models it is hard to predict to what extent the simplified model is able to estimate cost function gradients. One criteria would be the performance of the corresponding coarse resolution forward model for the estimation of the data that is assimilated. The adjoint is constructed by linearizing around the state from the high-resolution model which is assumed to increase the realism of the adjoint. The low unrealistic penetration scale of the jet stream of the coarse resolution model is thus less crucial.

In case of the Lorenz model the statistical moments were shown to be Gaussian distributed quantities. This condition enables the validity of the maximum likelihood argument used in variational methods which is generally found to be violated in highly nonlinear models (Miller et al., 1999). However, the restoration of this condition should not be over-rated, since its validity is limited by a time scale separation argument which probably will not hold for ocean models in same distinct way as in the Lorenz case.

The precision up to which parameters could be recovered is limited by the variability of statistical moments. The cost function gradient estimated by the method is only approximately correct and it is not clear how this error contributes to the final error in recovering the parameters. Both from the optimization and from a simple theoretical consideration, error values were found to be higher than $20 \%$, which at the first sight would not be very encouraging for future applications of the method in basin-wide ocean models. However, this is not a deficiency of the method, but results from very high variability of the averaged quantities in the most nonlinear region of western boundary currents. The estimation of parameter errors were less successful. This was due to large low-frequency variability of the stream function leading to a large subspace of equivalent parameter values. The basin-wide application that is subject of a second article shows lower noise level and greater precision for parameter estimations. Although the assimilation of variance only reached cost function values below the noise level almost without reducing the parameter error, the mean stream function is corrected simultaneously. The estimation of the mean position of the jet from variability only is well conditioned.

\section{Acknowledgments}

The work was supported by the Bundesministerium für Forschung und Technologie as part of the German WOCE. We would like to thank J. Schröter and A. Vogeler, who provided the QG model and the adjoint used in this study.

\section{REFERENCES}

Boffetta, G., Giuliani, P., Paladin, G. and Vulpiani, A. 1998. An extension of the Lyapunov analysis for the predictability problem. J. Atmos. Sci. 23, 3409-3416.

Courtier, P., Thépaut, J. N. and Hollingsworth, A. 1994. A strategy for operational implementation of 4D-Var, using an incremental approach. Q. J. R. Meteorol. Soc. 120, 1367-1387.

Eckmann, J.-P. 1981. Roads to turbulence in dissipative dynamical systems. Rev. Mod. Phys. 53, 643-654.

Evensen, G. 1992. Using the extended Kalman filter with a multilayer quasi-geostrophic ocean model. J. Geophys. Res. 97, 17,905-17,924.

Evensen, G. 1994. Sequential data assimilation with a nonlinear quasigeostrophic model using Monte Carlo methods to forecast error statistics. J. Geophys Res. 99, 10,143-10,162.

Evensen, G. and van Leeuwen, P. J. 1996. Assimilation of Geosat altimeter data for the Agulhas Current using the ensemble Kalman filter with a quasigeostrophic model. Mon. Wea. Rev. 124, 85-96.

Farge, M. 1992. Wavelet transforms and their applications to turbulence. Ann. Rev. Fluid. Mech. 24, 395-457.

Fox, A., Haines, K., de Cuevas, B. and Webb, D. 1998. Assimilation of TOPEX data into the OCCAM model. Int. WOCE Newsletter 31, 12-15.

Gauthier, P. 1992. Chaos and quadri-dimensional data

Tellus 54A (2002), 4 
assimilation: a study based on the Lorenz model. Tellus 44A, 2-17.

Green, J. S. A. 1970. Transfer properties of the largescale eddies and the general circulation of the atmosphere. Q. J. R. Meteorol. Soc. 96, 157-417.

Griffies, S. M. and Bryan, K. 1997. Predictability of North Atlantic multidecadal climate variability. Science 275, 181-184.

Holland, W. R. 1978. The role of mesoscale eddies in the general circulation of the ocean - Numerical experiments using a wind-driven quasi-geostrophic model. J. Phys. Oceanogr. 8, 363-392.

Holloway, G. and Hendershott, M. C. 1977. Stochastic closure for nonlinear Rossby waves. J. Fluid Mech. 82, 747-765.

Jung, T., Ruprecht, E. and Wagner, F. 1998. Determination of cloud liquid water path over the oceans from Special Sensor Microwave/Imager (SSM/I) data using neural networks. J. Applied Met. 37, 832-844.

Killworth, P. D., Dietrich, C., Provost, C. L., Oschlies, A. and Willebrand, J. 2001. Assimilation of altimetric data and mean sea surface height into an eddy-permitting model of the North Atlanic. Progr. Oceanogr. 48, 313-335.

Le Dimet, F.-X. and Talagrand, O. 1986. Variational algorithms for analysis and assimilation of meteorological observations: theoretical aspects. Tellus 38A, 97-110.

Lea, D. J., Myles, A. and Haine, T. W. N. 2000. Sensitivity analysis of the climate of a chaotic system. Tellus 52A, 523-532.

Li, Y. 1991. A note on the uniqueness problem of variational adjustment approach to four-dimensional data assimilation. J. Meteotol. Soc. Jpn. 69, 581-585.

Lorenz, E. N. 1963. Deterministic nonperiodic flow. J. Atmos. Sci. 20, 130-141.

Lorenz, E. N. 1975. Climate predictability: the physical basis of climate modeling. WMO, GARP Pub. Ser. 16, 132-136.

Marotzke, J. and Wunsch, C. 1993. Finding the steady state of a general circulation model through data assimilation: application to the North Atlantic Ocean. J. Geophys. Res. 98, 20,149-20,167.

Meacham, S. P. 2000. Low frequency variability in the wind-driven circulation. J. Phys. Oceanogr. 30, 269-293.

Miller, R. N., Carter, E. F. and Blue, S. T. 1999. Data assimilation into nonlinear stochastic models. Tellus 51A, 167-194.

Miller, R. N., Ghil, M. and Gauthiez, F. 1994. Advanced data assimilation in strongly nonlinear dynamical systems. J. Atmos. Sci. 51, 1037-1056.

Moore, A. M. 1991. Data assimilation in a quasi-geostrophic open-ocean model of the Gulf Stream region using the adjoint method. J. Phys. Oceanogr. 21, 398-427.

Nese, J. M., Dutton, J. A. and Wells, R. 1987. Calculated attractor dimensions for low-order spectral models. J. Atmos. Sci. 44, 1950-1972.

Oschlies, A. and Willebrand, J. 1996. Assimilation of Geosat altimeter data into an eddy-resolving primitive equation model of the North Atlantic Ocean. J. Geophys. Res. 101, 14,175-14,190.

Oseledec, V. I. 1968. A multiplicative ergodic theorem. Lyapunov characteristic numbers for dynamical systems. Trans. Moscow Math. Soc. 19, 197-231.

Palmer, T. N. 1993. Extended-range atmospheric prediction and the Lorenz model. Bull. Am. Meteorol. Soc. 74, 49-65.

Palmer, T. N. 1996. Predictability of the atmosphere and oceans: from days to decades. In: Decadal climate variability (eds. D. T. L. Anderson and J. Willebrand), NATO ASI Series 44, 83-156.

Pires, C., Vautard, R. and Talagrand, O. 1996. On extending the limits of variational assimilation in nonlinear chaotic systems. Tellus $\mathbf{4 8 A}, 96-121$.

Press, W. H., Teukolsky, S. A., Vetterling, W. T. and Flannery, B. P. 1993. Numerical recipes in Fortran. Cambridge University Press, $963 \mathrm{pp}$.

Rabier, F., Thépaut, J. N. and Courtier, P. 1998. Extended assimilation and forecast experiments with a four-dimensional variational assimilation system. Q. J. R. Meteorol. Soc. 124, 1861-1887.

Risken, H. 1984. The Fokker-Planck equation: methods of solution and applications. Springer-Verlag, Berlin, $545 \mathrm{pp}$.

Schiller, A. and Willebrand, J. 1995. The mean circulation of the Atlantic Ocean north of $30 \mathrm{~S}$ determined with the adjoint method applied to an ocean general circulation model. J. Marine Res. 53, 453-497.

Schröter, J., Seiler, U. and Wenzel, M. 1993. Variational assimilation of Geosat data into an eddy resolving model of the Gulf Stream extension area. J. Phys. Oceanogr. 23, 925-953.

Sirkes, Z. and Tziperman, E. 1997. Finite difference of adjoint or adjoint of finite difference? Mon. Wea. Rev 125, 3373-3378.

Stammer, D. 1997. Global characteristics of ocean variability estimated from regional TOPEX/POSEIDON altimeter measurements. J. Phys. Oceanogr. 27, 1743-1768.

Stensrud, D. J. and Bao, J. W. 1992. Behaviors of variational and nudging techniques with a chaotic loworder model. Mon. Wea. Rev. 120, 3016-3028.

Stone, P. H. 1972. A simplified radiative-dynamical model for the static stability of rotating atmospheres. J. Atmos. Sci. 29, 405-418.

Tanguay, M., Bartello, P. and Gauthier, P. 1995. Fourdimensional assimilation with a wide range of scales. Tellus 47A, 947-967.

Thacker, W. C. 1986. Relationships between statistical and deterministic methods of data assimilation. Variational methods in geosiences (ed. Y. K. Sasaki). Elsevier, Amsterdam.

Thacker, W. C. 1989. The role of the Hessian matrix in 
fitting models to measurements. J. Geophys. Res. 94, Yu, L. and Malanotte-Rizzoli, P. 1998. Inverse modeling $6177-6196$.

Yu, L. and Malanotte-Rizzoli, P. 1998. Inverse modeling

Vogeler, A. and Schröter, J. 1995. Assimilation of satellite J. Phys. Oceanogr. 28, 902-922.

altimeter data into an open ocean model. J. Geophys.

Res. 100, 15,951-15,963.

Tellus 54A (2002), 4 